
\title{
25 Research Soure \\ Psychological distress during the COVID-19 pandemic: The interplay between protective and risk factors
}

Serena Petrocchi ( $\sim$ serena.petrocchi@ieo.it )

Applied Research Division for Cognitive and Psychological Science, European Institute of Oncology IRCCS

\section{Paola lannello}

Department of Psychology, Catholic University of the Sacred Heart

\section{Giulia Ongaro}

Applied Research Division for Cognitive and Psychological Science, European Institute of Oncology IRCCS

\section{Alessandro Antonietti}

Department of Psychology, Catholic University of the Sacred Heart

\section{Gabriella Pravettoni}

Applied Research Division for Cognitive and Psychological Science, European Institute of Oncology IRCCS

\section{Research Article}

Keywords: risk aversion, ambiguity tolerance, COVID-19, psychological distress, information seeking, worry

Posted Date: September 29th, 2020

DOl: https://doi.org/10.21203/rs.3.rs-83524/v1

License: (c) (i) This work is licensed under a Creative Commons Attribution 4.0 International License. Read Full License 


\section{Abstract}

Background. Individuals under quarantine, such as the one caused by SARS-CoV-2, experience severe distress. The present research aimed to test a model of relations to ascertain the determinants of distress. Worry, perceived utility of the lockdown, and health-related information seeking were mediators in the relationships between COVID-19 exposure, risk aversion, and intolerance for ambiguity, on one side, and distress on the other.

Method. The study was conducted in Italy during the mandatory lockdown for the COVID-19 pandemic on 240 individuals (age range 18-76).

Results. COVID-19 exposure was positively associated with worry about COVID-19 and health-related information seeking; risk aversion was positively associated with health-related information seeking and perceived utility of the lockdown to contain the spread of the virus. Worry and health-related information seeking were positively associated with distress, while the perceived utility of the lockdown was negatively associated with distress. Intolerance for ambiguity was directly linked to distress with a positive sign.

Conclusions. Our evidence suggests that risk aversion represents both a risk factor and a protective factor based on what kind of variable mediates the relationship with distress. However, intolerance to ambiguity is a risk factor for buster distress.

\section{Background}

According to the European Center for Disease Prevention and Control (ECDC, 2020a) since 31 December $2019,8,000,847$ cases of COVID-19 have been reported worldwide, including 436,632 deaths. Caused by the virus SARS-CoV-2, the WHO has declared COVID-19 as a pandemic in March 2020. In Italy, in March and April, the time in which the present research was carried out, the official number of cases reached 110.574 with 13.157 deaths. In the same period, in the U.S. The cases were 216.721, 102.136 in Spain, 73.522 in Germany, 56.989 in France, and 29.474 in the United Kingdom (ECDC, 2020b). In response to the increasing number of infections and deaths, European countries have implemented nonpharmaceutical measures, including social distancing, border closures, school closures, isolation measures for symptomatic individuals and their contacts, and the lockdown of the population with internal travel banned. In Italy, the lockdown started on March 8 and ended on May 18 and involved almost the entire population.

Lockdown is effective in quickly reducing the spread of the virus (Nussbaumer-Streit et al., 2020). However, a rapid review of the studies carried out during previous pandemics (e.g., SARS, Ebola, H1N1 influenza) revealed a negative psychological impact generated by physical isolation and quarantine (Brooks et al., 2020; Rubin and Wessely, 2020). Short-term effects involved emotional disorders, anxiety, depression, stress, mood decline, irritability, insomnia, and PTSD. Another umbrella review (Hossain et al., 2020) reported a high burden of mental health conditions among individuals who experienced 
quarantine, including anxiety, depression, anger, irritability, and psychosocial distress. Evidence suggests that such impacts continued over a long period, after the end of the lockdown, highlighting that acute exposure to psychosocial stressors during quarantine and social isolation can exert prolonged impacts on psychological functioning and mental health (Park and Park, 2020; Shigemura et al., 2020). What is still to be identified is the determinants of mental distress and the mechanisms underlying it. The present study aimed to analyze several mechanisms to detect both protective and risk factors in predicting distress during lockdown due to the COVID-19 outbreak.

One first main, and perhaps trivial, variable that may influence individuals' distress is direct exposure to COVID-19. It is reasonable to assume that the more individuals are exposed to a physical threat, the more they perceive high health risk for themselves and significant others (Cava et al., 2005) and then the more they experience distress. However, other variables may play a role in this relationship. Hawryluck and colleagues (2004) showed that acquaintance with or direct exposure to someone with SARS diagnosis was associated with a worrying condition. Worry is a cognitive coping response that serves as an avoidant function to respond to the immediate distress associated with a threat or something that could be perceived as potentially overwhelming and dangerous (Borkovec et al., 2004). However, paradoxically, this strategy in the long term may lead to increased distress (Hayes et al., 1996), anxiety, and mood disorders (Beck et al., 2001).

Furthermore, there is evidence that during outbreaks of epidemic or pandemic proportions, people require immediate health information (Gudykunst and Nishida, 2001; Hyvärinen and Vos, 2016). When experiencing fear, people are pushed toward seeking information (Allen et al., 2014; Lin et al., 2014; blind for review), given that search can help mitigate the feelings of uncertainty that generally spread during a time of threat (Kossowska and Bukowski, 2015). For example, immediately following the announcement of the H1N1 outbreak, people increased their health-information seeking behaviors on websites (Tausczik et al., 2012). Although information seeking behavior is one of the possible responses to uncertainty (Rosen et al., 2007; Rosen and Knäuper, 2009), it may result in an increased level of distress (Rosen et al., 2007), especially when the information gathered is inconsistent (Cava et al., 2005). Finally, research has started to suggest that the willingness to adhere to quarantine and lockdown measures is more prominent in individuals perceiving the threat as more dangerous (Dryhurst et al., 2020) and among those who have pronounced prosocial traits (Pfattheichera et al., 2020). As a result of this association, those individuals might also experience a better adaptation to the measures of restriction of freedom and then better mental health (see blind for review). Therefore, following the abovementioned considerations, we conjectured the following:

Hypothesis 1 - Exposure to the COVID-19 increases distress directly (HP1a) and indirectly through the mediation of higher perception of worry (HP1b), greater health-related information seeking (HP1c), and better perception of the utility of the lockdown as a measure to decrease the COVID-19 spread (HP1d).

In addition to contingent and external factors, such as exposure to COVID-19, other factors might influence individuals' level of distress during pandemic. The literature indicates that individual differences 
in the ability to tolerate ambiguous situations (e.g., situations that "cannot be adequately structured or categorized by an individual because of the lack of sufficient cues"; Budner, 1962: p. 30) might influence the level of distress perceived by individuals. Specifically, when faced with unclear and unstructured situations, individuals with low tolerance for ambiguity perceive them as a source of discomfort and threat, and this tendency to react negatively to ambiguity and uncertainty is likely to lead to an increased level of distress (Furnham and Ribchester, 1995; blind for review; Mac Donald, 1970; McLain, 1993; Budner, 1962), anxiety (Bardi et al., 2009), and worry (Buhr and Dugas, 2006). Furthermore, among ambiguity intolerant people, high levels of worry are also associated with the lack of perceived control over the situation (Endler et al., 2000). Individuals characterized by low tolerance for ambiguity might decide to behave in a way that they consider to be protective, such as seeking health information as a way to reduce the level of uncertainty due to the COVID-19 pandemic and adhering to the lockdown restriction as a protective way to respond to the spread of the COVID-19 outbreak.

Therefore, in the context of profound uncertainty and ambiguity, such as the COVID-19 pandemic outbreak, where future conditions are largely unpredictable and consistent information is mostly lacking, we expected the following:

Hypothesis 2 - Higher levels of ambiguity intolerance correspond to higher distress directly and through the mediation of higher perception of worry (HP2b), greater health information seeking behaviors (HP2c), as a way to overcome uncertainty-induced discomfort, and higher perceived utility of the lockdown as a measure of control of the pandemic (HP2d).

The literature highlights the existence of a relationship between intolerance for ambiguity and risk-taking propensity (Caligiuri and Tarique, 2012). Individuals' reactions to risk and ambiguity tend to be consistent (Lauriola et al., 2007). In addition to ambiguity intolerance, there appears to be an association between the perception of risk and the tendency to worry. Risk attitude has been defined as a dispositional trait or characteristic that reflects a "person's standing on the continuum from risk aversion to risk seeking" (Weber et al., 2002: p. 264). The literature has found that greater risk aversion is generally associated with the tendency to worry and anxiety (Lorian and Grisham, 2011; Maner and Schmidt, 2006). The tendency to overestimate the probability and severity of negative outcomes could possibly contribute to explaining the relationship between negative risk orientation and worry (Koerner et al., 2017). Furthermore, risk attitude has been found to be a motivator for information-seeking behaviors (Griffin et al., 2004; Turner et al., 2006) and a predictor of self-protective actions (Janz and Becker, 1984). Perceiving a heightened likelihood of aversive events may push risk-avert people to seek out information and take preventive action to control the situation, especially during an emergency, such as the COVID-19 pandemic. Therefore, we expected the following:

Hypothesis 3 - Risk aversion positively influences individuals' distress directly (HP3a) and indirectly through increasing worry (HP3b), greater health-related information seeking behavior (HP3c), and more positive perception of the utility of the lockdown as a useful way to keep the spread of the COVID-19 under control (HP3d). 
The hypothesized associations among constructs are summarized in a theoretical model shown in Figure 1. Given that women experience higher distress than men (Papini et al., 1990), gender has been tested as a covariate.

[Figure 1 about here ]

\section{Methods}

\section{Participants}

The study was conducted in Italy during March-April 2020 using a snowball sampling method by posting the link to the survey on several public and private Facebook pages. The survey was implemented on Qualtrics ${ }^{\mathrm{XM}}$ software. The inclusion criteria were age $18+$ and fluency in Italy and residence in Italy. Health-related workers (physicians, nurses, psychiatrists, psychologists, and psychotherapists) were excluded from the sample because they were not forced to respect the lockdown. An a priori power analysis applying G*Power 3.1.9.4 (Faul et al., 2007) was conducted to determine the sample size (with alevel $=.05$, power $=.95, \eta^{2}=0.07$ ), and the final estimate was 224 participants.

Participants were 240 adults from 18 to 76 years of age $(M=39.67, S D=15.2 ; 79$ males $)$. Most of them were employed (55\%), 37\% were not employed, and $8 \%$ were retired. One hundred eighty-six participants declared to be in a stable relationship (78\%), whereas $8 \%$ lived alone at the time of the data collection (92\% lived with at least one family member).

\section{Measures}

A self-administered questionnaire was created to assess sociodemographic characteristics to collect data on gender, age, employment, relationship status, and family size.

COVID-19 Exposure. Participants were asked to indicate whether they tested positive or experienced symptoms related to the COVID-19. Similarly, they were asked whether their partner, relatives or friends tested positive or had had compatible symptoms (Li et al., 2020). Response options were dichotomous, and a final score was computed as a sum. The higher the final score, the higher the number of exposures of the participant to COVID-19 infection, to people infected, or with compatible symptoms with COVID-19.

Worry. Participants were asked to indicate their worry about contracting COVID-19 infection by themselves, family members, and significant others with 3 items on a 5-point scale (ranging from "strongly disagree" to "strongly agree"). A sample item is "I feel worried that I may be infected with COVID19". A higher final score indicates that participants had high worries about COVID-19 infection. In this study, this scale has been shown to be internally consistent $(a=.82$, rs $>.51)$.

Health-related information seeking. The frequency with which participants searched medical information through physicians during COVID-19 lockdown was investigated with a single item. A 5-point Likert scale 
ranging from "never" to "ever" was used, with higher scores indicating a higher tendency to seek information.

Utility of the Lockdown. Participants were asked to indicate their perceived utility of the lockdown to stop spreading COVID-19. It was evaluated with 3 items on a 5-point scale ranging from "strongly false" to "strongly true". A sample item is "Quarantine is not very effective to stop the spread of COVID-19" (reversed). A higher score is indicative of the perception of greater usefulness of the lockdown as a measure of outbreak containment. The internal consistency of the scale was moderate $(a=.70, r s>.43)$.

Risk aversion. This construct was measured with the Health Risk Attitude Scale (HRA-6; Dieteren et al., 2020), a self-report scale (6 items) that assesses how a person would resolve risky health decisions and includes questions related to risky and preventive health behavior and related to medical treatment. Ranging from "completely disagree" to "completely agree", the 5-point Likert scale is used to measure the individual health risk attitude. A total score was calculated with a higher score indicating a stronger riskaverse attitude. In this study, it has been shown to be internally consistent (Cronbach $a=.79$, rs > .17, CFA results $\chi^{2}(9)=30.85, p<.001, \mathrm{CFI}=.96$, RMSEA $\left.=.10\right)$.

Intolerance for ambiguity. The construct was measured with the ambiguity scale of the Need for Closure questionnaire (Pierro et al., 1995). The intolerance for ambiguity is measured through 7 items that are defined as a desire for any type of answer to end further information processing, rather than remaining in doubt and ambiguity. Response options varied from 1 ("strongly disagree") to 4 ("agree"). Cronbach's alpha coefficients were equal to .68 for the total scale (with the exclusion of item 7; rs > .31; CFA results $\chi^{2}$ $(14)=44.49, \mathrm{p}<.001, \mathrm{CFI}=.85, \mathrm{RSMEA}=.09)$.

Distress about the COVID-19 outbreak. Emotional distress related to COVID-19 was investigated by asking participants how often they felt a specific emotional reaction (calm, tense, upset, relaxed, concerned, uninvolved, content, and active) on a 4-point Likert scale during the previous two weeks. A reverse score was applied to the positive emotions, and a total score was calculated as the average. A higher total score indicates higher distress thinking to the health emergency related to COVID-19. In this study, the scale showed Cronbach's alpha coefficients equal to .80 ( $r s>.25$; CFA results $\chi^{2}(14)=61.34, p<.001$, CFI $=90$, RSMEA $=.10)$.

\section{Procedure}

Data collection was conducted via snowball sampling. The survey was spread through a Qualtrics link. The only inclusion criterion was being adults. The exclusion criterion was having a job as health-care professionals to keep under control the individual's level of engagement in COVID-19 management. Participants completed informed consent before starting the survey and did not receive any compensation. Ethical approval was obtained from the University's Ethical Committee (N. 19133).

\section{Data Analysis Strategy}


Data analyses were performed using the statistical software analysis package SPSS (Version 26.0) and AMOS v. 24. Data were normally distributed. Independent samples t-tests were performed to identify systematic differences in the distribution of gender in the variables. Correlations were executed to test the association between variables. For the main analyses, the hypothesized model (Figure 1) was tested using structural equation modeling (SEM). Gender was entered as a control variable for distress. The following goodness-of-fit indices were used to evaluate model-data correspondence: $\chi^{2}$ value, Comparative Fit Index (CFI), and Root Mean Square Error of Approximation (RMSEA). Given that the $\chi^{2}$ value is influenced by the sample size, Byrne (2016) suggested accepting a model when the CFI is higher than .90 and close to .95 and the RMSEA is .08 or less. Finally, modification indices and the matrix of standardized correlation residuals were inspected for potential improvement of model fit. The reverse model was tested and compared to the main model via $\Delta \chi^{2}$.

\section{Results}

\section{Preliminary analyses}

T-tests showed a significant difference between males and females regarding the average of the scores in the perceived usefulness of the lockdown $(t(113.567)=-2.130, p=.035)$. Specifically, women perceived the lockdown more useful $(M=4.36 ; S D=.669)$ than that perceived by men $(M=4.10 ; S D=.888)$. Women also showed, on average, higher levels of distress $(M=2.99, S D=.41)$ than those manifested by men $(M=2.75 ; S D=.50)$. This difference was significant $(t(131.321)=-3.704, p<.001)$. No other statistically significant differences were found between males and females in the dimensions belonging to the model presented.

Significant associations emerged between gender, utility of the lockdown, and distress. Age correlated with utility of the lockdown. Family size with COVID-19 exposure, which, in turn, correlated with worry and health-related information seeking. Risk aversion correlated with utility of the lockdown. Ambiguity correlated with distress, which was correlated with worry, health-related information seeking, and utility of the lockdown. Table 1 shows the means, standard deviations, and correlations between variables.

[insert table 1 approx. here]

\section{Primary analyses testing the hypothesized model}

The hypothesized model was tested with distress on the total sample $(n=240)$. Gender was added as a control variable. The final model (Figure 2) showed a good fit of the data with $\chi^{2}(17)=15.48, p=.56, \mathrm{CFI}$ $=1$, and RMSEA $=.00,90 \% \mathrm{Cl}[.000 .054]$. Examination of path coefficients yielded evidence for the hypothesized paths.

<<Figure 2 about here > 
Worry was significantly related to distress $(\beta=.31, p<.001)$, indicating that increased worry for COVID-19 led to higher levels of distress during quarantine. Likewise, the utility of the quarantine was significantly negatively related to distress $(\beta=-.24, p<.001)$. Information-seeking behavior was significantly related to distress $(\beta=.13, p=.033)$, i.e., the more an individual asks for information to a doctor during the quarantine, the more he experiences distress. Furthermore, information seeking behavior and utility of the quarantine were correlated with each other $(\beta=.15, p=.025)$. Among the predictors, COVID-19 exposure was significantly related to worry $(\beta=.23, p<.001)$ and information seeking behavior $(\beta=.18, p=.006)$, whereas risk aversion was significantly related to information seeking behavior $(\beta=.20, p=.003)$ and utility of the quarantine $(\beta=.29, p<.001)$.

Moreover, the indirect paths from COVID-19 exposure to distress were significant both through worry ( $\beta=$ $.03, p<.001)$ and health-related information seeking $(\beta=.011, p=.008)$, while the indirect paths from risk aversion to distress through health-related information seeking and utility of the lockdown were not significant. The total effect from COVID-19 exposure to distress and from risk aversion to distress were not significant. Additionally, gender was significantly related to distress $(\beta=.21, p<.001)$, with females being more stressed than males.

[insert Figure 2 approx. here]

Since the present research has a cross-sectional design, the reverse model was also tested, reaching a significant $\left.\chi^{2}(21)=42.34, p=.004\right)$ and worse $C F I=.78$, RMSEA $=.065$ compared to the previous model. $\Delta \chi^{2}(4)=26.86, p<.001, \triangle C F I$ and $\triangle R M S E A=.065$ indicate that the first model better fit the data.

\section{Discussion}

The non-pharmaceutical measures implemented during the COVID-19 pandemic, such as the lockdown of the population and quarantine of positive cases, have been found to be effective in quickly reducing the spread of the virus (Nussbaumer-Streit et al., 2020). However, lockdown is often an unpleasant experience for those who undergo it. Separation from loved ones, loss of freedom, uncertainty over disease status and the duration of the lockdown itself, burden for financial and economic consequences, and boredom can, on occasion, create dramatic effects (Brooks et al., 2020; Hossain et al., 2020). The eventuality of a second wave of COVID-19 was already hypothesized in April 2020 (Leung et al., 2020), and then many hotspots were confirmed, for example, in Italy, China, and Germany in the subsequent months. Massive efforts have been applied by public health interventions nationwide to contain outbreaks. However, psychological supportive interventions are particularly needed to help people cope with the situation (Dominguez-Salas et al., 2020; Lei et al., 2020). The present study aimed to examine several mechanisms responsible for high levels of distress and then to identify possible mechanisms to be considered in supportive interventions. In particular, we studied the impact of two individual features, risk aversion and intolerance of ambiguity, together with COVID-19 exposure, on distress measured during the mandatory lockdown in Italy. These relations were tested through the moderation of worry, health-related information seeking behavior, and perception of the utility of the lockdown. 
Research has demonstrated that being directly exposed to COVID-19 threat is associated with physical symptoms, stress, and anxiety (Chew et al., 2020; Wang et al., 2020). The exposure of a serious threat to physical safety enhances the perception of health risk for themselves and for significant others (Cava et al., 2005) and, consequently, distress. In line with such considerations, our evidence suggests that COVID19 exposure increased worry perception (blind for review), which, in turn, was associated with higher distress. This result is consistent with the literature (Gudykunst and Nishida, 2001), which identifies high uncertainty as a risk factor for the development of negative psychological mental health. COVID-19 exposure brings people in direct contact with high levels of uncertainty due to possible serious medical complications and lack of effective and specific treatments for the virus. Similarly, COVID-19 exposure enhances health-related information seeking as a way to limit uncertainty due to the pandemic, and this leads to greater distress for reasons that we explore just below.

In addition to COVID-19 exposure, our results suggested that individual characteristics exert an effect on individual psychological well-being. Risk aversion determined greater health-related information seeking and perception of utility of the lockdown, which were significantly related to distress with a positive and negative sign, respectively. Therefore, our results provide an articulated picture. It seems that risk aversion represented a risk factor when stimulating health information seeking because, in an uncertain situation characterized by informative overload (Reich et al., 2006), the search for information leads to worse psychological functioning. Even though information seeking behavior is one of the possible responses to uncertainty (Rosen et al., 2007; Rosen and Knäuper, 2009), it may also result in an increased level of distress (Reich et al., 2006), depending on the specific content of the information (Rosen et al., 2007). This aspect was also explained by Cava and colleagues (2005) during the SARS outbreak, when many participants reported that inconsistent information from various sources led them to question the reliability of available information. Moreover, as shown by others (Dominguez-Salas et al., 2020), when adequate information is lacking, people double their efforts to fill the informative gap, sometimes choosing non-reliable sources such as social media and internet (Abd-Alrazaq et al., 2020; Cuan-Baltazar et al., 2020; Zhao et al., 2020) and contributing to creating overload. Therefore, during the COVID-19 lockdown, a condition in itself characterized by profound uncertainty, incomplete and inconsistent information, even if received by physician, together with an informative overload may have cast doubt on the reliability of information and on the trust placed in the health professionals, consequently generating higher levels of distress.

On the other hand, risk aversion acted as a protective factor increasing the perception of utility of the lockdown. When individuals think that the lockdown is effective in reducing the spread of the pandemic, although it interrupts normal daily functioning and has a detrimental effect on their psychological adaptation (Brooks et al., 2020), they might be able to adapt themselves to the mandatory situation, leading to lower distress.

Contrary to the expectations, worry did not mediate the relationship between risk aversion and distress. This result may probably be related to the specific scale used in the present study to measure the construct of worry. The literature reporting an association between risk aversion and worry mainly 
referred to trait and generalized excessive worry (Lorian and Grisham, 2011). Our items specifically measured the level of worry that people perceived in responses to the COVID-19 outbreak because that was the focus of our research. This may have reduced the strength of the association.

Finally, intolerance for ambiguity was found to be directly associated with distress, which was higher in females than males. Therefore, it seems that the higher the ability to tolerate ambiguity, the less emotional distress experienced, in a specific situation where uncertainty and unpredictability dominate, as during the lockdown due to COVID outbreak. Regardless, the relationships between low tolerance for ambiguity and decision-making are not linear. Intolerance for ambiguity may generate behaviors aimed at reducing the level of uncertainty even if, on a long time basis, such behaviors might no longer be adaptive or appropriate and might enhance a negative emotional experience (Han et al., 2006, 2007). Ambiguity aversion thus leads to an alarmistic response, a pessimistic bias (Viscusi, 1997) defined as the tendency to reserve an excessive amount of attention and effort to the worst scenario possible in case of ambiguous risk information. The pessimistic bias has been proven to be persistent over time even when the situation is less far than ambiguous (Curley and Yates, 1985; Keren and Gerritsen, 1999) and able to provoke a sequela of psychological symptoms.

Worry, health-related information seeking, and perceived utility of the lockdown did not mediate the relation between low tolerance for ambiguity and distress. For worry, an association was expected since the literature found that trait worry increases in individuals with a low level of tolerance of ambiguity (Buhr and Dugas, 2006). In our research, as mentioned before, we measured a state worry dimension specifically referred to the COVID-19 spread, which might have deflated the expected association, as for risk aversion.

For health-related information seeking behavior, we expected to find a relationship between a high level of ambiguity intolerance and the tendency to seek out information (Litman, 2010) as a way to obtain greater control over the situation. However, it has to be taken into consideration the peculiarity of the COVID-19 situation, defined as "infodemic" (WHO, 2020), thus referring to the overabundance of information, often inconsistent, fragmented, incomplete and not accurate. High ambiguity intolerant people, who generally find it difficult to manage contradictions and incomplete information (Chiang, 2016), may have experienced a sense of confusion and overload that have prevented them from seeking out further information. It might be that high ambiguity intolerant people, when overwhelmed by numerous and incoherent information, struggle to integrate them in a coherent picture and then tend to avoid searching for other information. Finally, high ambiguity aversion, combined with the amount of information received by individuals, might have decreased trust toward institutions (Samson \& ostyszyn, 2015; Zhou et al., 2017) and, by extension, toward the restrictive measures they imposed (e.g., lockdown).

\section{Limitations}

The present study has several limitations. First, most of our participants (or their partner, family members, friends) were not directly exposed to COVID-19. Although status reflects the situation of the majority of 
the people under lockdown due to the COVID-19 pandemic, different paths of relations among variables could be expected if COVID-19-positive individuals would have been interviewed. For many evident practical and ethical reasons, data collection on COVID-19-positive individuals would be possible only retrospectively. A second limitation concerns the measures applied in the study. All the measures we utilized are self-reported, and distress items have been created ad hoc for the study. We realize that this might have created a bias in the results. However, during the lockdown period, it was impossible to conduct data collection via non-self-reported measures, and given the urgency of the investigation, we chose for the faster and quicker way possible. Finally, we treated the ambiguity of intolerance in the context of the need for closure using the discomfort with ambiguity subscale of the Need for Closure questionnaire. Even if that subscale correlates with other questionnaires measuring ambiguity intolerance (e.g., Webster and Kruglanski, 1994), we should be cautious in generalizing the results. Third, we did not differentiate among different sources of stress. We asked participants to think about the previous weeks when replying to the questions regarding the emotional experience. However, it might be that the source of the distress would come from events other than the COVID-19 pandemic but related to it, such as difficulties in balancing smart working and family responsibilities, financial loss, or the risk of losing the job.

\section{Conclusion}

Our research highlights the role played by several factors in moderating the levels of distress during an extremely challenging situation, such as the one people experiment during a pandemic and a subsequent lockdown period. Our evidence suggests the presence of differential paths between risk aversion and distress and between intolerance for ambiguity and distress. Risk aversion represents, at the same time, a risk and a protective factor based on what variable mediates its relationship with distress. This evidence suggests that risk aversion might have a differential effect on individuals' decision-making and behaviors and then on distress. On the other hand, intolerance for ambiguity is directly linked to distress and thus represents a risk factor for mental health. Taken together, this evidence leads to some considerations regarding the possible interventions sustaining individuals' mental health and the principles guiding communication in time of crisis.

Supporting individuals' psychological adjustment under the urgency of a pandemic is quite challenging, but several attempts are ongoing (Bashshur et al., 2020; Bäuerle et al., 2020). Interventions aiming to diminish distress by reducing the negative effect of risk factors and activating protective factors (Bäuerle et al., 2020) may find new support for our results. Our findings support the evidence that individuals' variables, together with COVID-19 exposure, play a role in mental health during a pandemic outbreak.

Furthermore, balanced communication during a health crisis is complex (blind for review), with social media and internet often provoking shocking or even false information. For the particular case of the COVID-19, communication has involved many channels (e.g., newscasts, trash TV programs, social media, internet), with different levels of competence and credibility. Our results suggest that pushing on the button of fear and uncertainty may lead to inconsistent outcomes and often have a boomerang effect 
(Witte, 1992) because low tolerance for ambiguity and greater worry lead to high distress and these aspects make people less willing to adopt protective behaviors (Ritov and Baron, 1990; Viscusi, 1997; Viscusi et al., 1991). Moreover, during a pandemic, the flow of information has to be controlled in its quantity, not only in its quality, because individuals with high risk aversion tendencies are more prone to look for information, and this behavior tends to enhance their distress.

\section{Declarations}

\section{Conflict of interest:}

The authors declare no competing interests.

\section{References}

Abd-Alrazaq A, Alhuwail D, Househ M, et al. (2020) Top concerns of tweeters during the COVID-19 pandemic: A surveillance study. Journal of Medical Internet Research. 22(4), e19016. DOI: $10.2196 / 19016$.

Allen DK, Karanasios S \& Norman A (2014) Information sharing and interoperability: The case of major incident management. European Journal of Information Systems. 23(4), 418-432. DOI: 10.1057/ejis.2013.8.

Bardi A, Guerra VM \&Ramdeny GSD (2009) Openness and ambiguity intolerance: Their differential relations to well-being in the context of an academic life transition. Personality and Individual Differences. 47(3), 219-223. DOI: 10.1016/j.paid.2009.03.003.

Bashshur R, Doarn CR, Frenk JM, et al. (2020) Telemedicine and the COVID-19 pandemic, lessons for the future. Telemedicine and e-Health. DOI: 10.1089/tmj.2020.29040.rb.

Bäuerle A, Graf J, Jansen C, et al. (2020) An e-mental health intervention to support burdened people in times of the COVID-19 pandemic: CoPE It. Journal of Public Health. DOI: 10.1093/pubmed/fdaa058.

Beck R, Perkins TS, Holder R, et al. (2001) The cognitive and emotional phenomenology of depression and anxiety: Are worry and hopelessness the cognitive correlates of NA and PA? Cognitive Therapy and Research. 25(6), 829-838. DOI: 10.1023/A:1012983726272.

Borkovec TD, Alcaine OM \& Behar E (2004) Avoidance Theory of Worry and Generalized Anxiety Disorder. Generalized Anxiety Disorder: Advances in Research and Practice.

Brooks SK, Webster RK, Smith LE, et al. (2020) The psychological impact of quarantine and how to reduce it: rapid review of the evidence. The Lancet. 395, 912-20. DOI: 10.1016/S0140-6736(20)30460-8. 
Budner S (1962) Intolerance of ambiguity as a personality variable. Journal of Personality. DOI: 10.1111/j.1467-6494.1962.tb02303.x.

Buhr K \& Dugas MJ (2006) Investigating the construct validity of intolerance of uncertainty and its unique relationship with worry. Journal of Anxiety Disorders. 20(2), 222-236. DOI: 10.1016/j.janxdis.2004.12.004.

Caligiuri P \& Tarique I (2012) Dynamic cross-cultural competencies and global leadership effectiveness. Journal of World Business. 47(4), 612-622. DOI: 10.1016/j.jwb.2012.01.014.

Cava MA, Fay KE, Beanlands HJ, et al. (2005) Risk perception and compliance with quarantine during the SARS outbreak. Journal of Nursing Scholarship. 37(4), 343-347. DOI: 10.1111/j.1547-5069.2005.00059.x.

Chew NWS, Lee GKH, Tan BYQ, et al. (2020) A multinational, multicentre study on the psychological outcomes and associated physical symptoms amongst healthcare workers during COVID-19 outbreak. Brain, Behavior, and Immunity. S0889-1591(20)30523-7. DOI: 10.1016/j.bbi.2020.04.049.

Chiang H-H (2016) A Study of Interactions among Ambiguity Tolerance, Classroom Work Styles, and English Proficiency. English Language Teaching. 9(6), 61-75. DOI: 10.5539/elt.v9n6p61.

Cuan-Baltazar JY, Muñoz-Perez MJ, Robledo-Vega C, et al. (2020) Misinformation of COVID-19 on the Internet: Infodemiology Study. JMIR Public Health and Surveillance. 6(2), e18444. DOI: 10.2196/18444.

Curley SP \& Yates JF (1985) The center and range of the probability interval as factors affecting ambiguity preferences. Organizational Behavior and Human Decision Processes. 36(2), 273-287. DOI: 10.1016/0749-5978(85)90016-0.

Dieteren CM, Brouwer WBF \& Van Exel J (2020) How do combinations of unhealthy behaviors relate to attitudinal factors and subjective health among the adult population in the Netherlands? BMC Public Health. 20, 1-14. DOI: 10.1186/s12889-020-8429-y.

Dominguez-Salas S, Gomez-Salgado J, Andres-Villas M, et al. (2020) Psycho-Emotional Approach to the Psychological Distress Related to the COVID-19 Pandemic in Spain: A Cross-Sectional Observational Study. Healthcare (Basel, Switzerland). 8(3),190.DOI: 10.3390/healthcare 8030190.

Dryhurst S, Schneider CR, Kerr J, et al. (2020) Risk perceptions of COVID-19 around the world. Journal of Risk Research. 1-13. DOI: 10.1080/13669877.2020.1758193.

European Centre for Disease Prevention and Control (ECDC) (2020a) COVID-19 situation update worldwide, as of 9 July 2020. Available at: https://www.ecdc.europa.eu/en/geographical-distribution2019-ncov-cases (accessed April 2, 2020).

European Centre for Disease Prevention and Control (ECDC) (2020b) Download today's data on the geographic distribution of COVID-19 cases worldwide. Available at: 
https://www.ecdc.europa.eu/en/publications-data/download-todays-data-geographic-distribution-covid19-cases-worldwide (accessed April 2, 2020).

Endler NS, Macrodimitris SD \& Kocovski NL (2000) Depression: The complexity of self-report measures. Journal of Applied Biobehavioral Research. 5(1), 26-46. DOI: 10.1111/j.1751-9861.2000.tb00062.x.

Faul F, Erdfelder E, Lang AG, et al. (2007) G*Power 3: A flexible statistical power analysis program for the social, behavioral, and biomedical sciences. Behavior Research Methods. 39(2), 175-191. DOI:

10.3758/BF03193146.

Furnham A \& Ribchester T (1995) Tolerance of ambiguity: A review of the concept, its measurement and applications. Current Psychology. 14(3), 179-199. DOI: 10.1007/BF02686907.

Griffin RJ, Neuwirth K, Dunwoody S, et al. (2004) Information Sufficiency and Risk Communication. Media Psychology. 6(1), 23-61. DOI: 10.1207/s1532785xmep0601_2.

Gudykunst WB \& Nishida T (2001) Anxiety, uncertainty, and perceived effectiveness of communication across relationships and cultures. International Journal of Intercultural Relations. 370(9590), 859-877. DOI: 10.1016/S0147-1767(00)00042-0.

Han PKJ, Moser RP \& Klein WMP (2006) Perceived ambiguity about cancer prevention recommendations: Relationship to perceptions of cancer preventability, risk, and worry. Journal of Health Communication. 11(S1), 51-69. DOI: 10.1080/10810730600637541.

Han PKJ, Moser RP \& Klein WMP (2007) Perceived ambiguity about cancer prevention recommendations: Associations with cancer-related perceptions and behaviours in a US population survey. Health Expectations. 10(4), 321-336. DOI: 10.1111/j.1369-7625.2007.00456.x.

Hawryluck L, Gold WL, Robinson S, et al. (2004) SARS control and psychological effects of quarantine, Toronto, Canada. Emerging Infectious Diseases. 10(7), 1206. DOI: 10.3201/eid1007.030703.

Hayes SC, Wilson KG, Gifford E V., et al. (1996) Experiential avoidance and behavioral disorders: A functional dimensional approach to diagnosis and treatment. Journal of Consulting and Clinical Psychology. 64(6), 1152. DOI: 10.1037/0022-006X.64.6.1152.

Hossain MM, Sultana A \& Purohit N (2020) Mental Health Outcomes of Quarantine and Isolation for Infection Prevention: A Systematic Umbrella Review of the Global Evidence. SSRN Electronic Journal. DOI: 10.2139/ssrn.3561265.

Hyvärinen J \& Vos M (2016) Communication Concerning Disasters and Pandemics: Coproducing Community Resilience and Crisis Response. The Handbook of International Crisis Communication Research. 43, 96. DOI: 10.1002/9781118516812.ch10. 
Janz NK \& Becker MH (1984) The Health Belief Model: A Decade Later. Health Education \& Behavior. 11(1), 1-47. DOI: 10.1177/109019818401100101.

Keren G \& Gerritsen LEM (1999) On the robustness and possible accounts of ambiguity aversion. Acta Psychologica. 103, 149-172. DOI: 10.1016/s0001-6918(99)00034-7.

Koerner N, Mejia T \& Kusec A (2017) What's in a name? Intolerance of uncertainty, other uncertaintyrelevant constructs, and their differential relations to worry and generalized anxiety disorder. Cognitive Behaviour Therapy. 46(2), 141-161. DOI: 10.1080/16506073.2016.1211172.

Kossowska M \& Bukowski M (2015) Motivated Roots of Conspiracies: The Role of Certainty and Control Motives in Conspiracy Thinking. The psychology of conspiracy.

Lauriola M, Levin IP \& Hart SS (2007) Common and distinct factors in decision making under ambiguity and risk: A psychometric study of individual differences. Organizational Behavior and Human Decision Processes. 104(2), 130-149. DOI: 10.1016/j.obhdp.2007.04.001.

Lei L, Huang X, Zhang S, et al. (2020) Comparison of Prevalence and Associated Factors of Anxiety and Depression among People Affected by versus People Unaffected by Quarantine during the COVID-19 Epidemic in Southwestern China. Medical Science Monitor. 26, e924609-1. DOI: 10.12659/MSM.924609.

Leung K, Wu JT, Liu D, et al. (2020) First-wave COVID-19 transmissibility and severity in China outside Hubei after control measures, and second-wave scenario planning: a modelling impact assessment. The Lancet. DOI: 10.1016/S0140-6736(20)30746-7.

Lin L, Savoia E, Agboola F, et al. (2014) What have we learned about communication inequalities during the H1N1 pandemic: A systematic review of the literature. BMC Public Health. 14(1), 484. DOI: 10.1186/1471-2458-14-484.

Litman JA (2010) Relationships between measures of I- and D-type curiosity, ambiguity tolerance, and need for closure: An initial test of the wanting-liking model of information-seeking. Personality and Individual Differences. 48(4), 397-402. DOI: 10.1016/j.paid.2009.11.005.

Lorian CN \& Grisham JR (2011) Clinical implications of risk aversion: An online study of risk-avoidance and treatment utilization in pathological anxiety. Journal of Anxiety Disorders. 25(6), 840-848. DOI: 10.1016/j.janxdis.2011.04.008.

Mac Donald AP (1970) Revised Scale for Ambiguity Tolerance: Reliability and Validity. Psychological Reports. 26(3), 791-798. DOI: 10.2466/pr0.1970.26.3.791.

Maner JK \& Schmidt NB (2006) The Role of Risk Avoidance in Anxiety. Behavior Therapy. 37(2), 181-189. DOI: 10.1016/j.beth.2005.11.003. 
McLain DL (1993) The mstat-i: A new measure of an individual's tolerance for ambiguity. Educational and Psychological Measurement. 53(1), 183-189. DOI: 10.1177/0013164493053001020.

Nussbaumer-Streit B, Mayr V, Dobrescu Al, et al. (2020) Quarantine alone or in combination with other public health measures to control COVID-19: a rapid review. The Cochrane database of systematic reviews. 4(4):CD013574. DOI: 10.1002/14651858.CD013574.

Papini DR, Farmer FF, Clark SM, et al. (1990) Early adolescent age and gender differences in patterns of emotional self-disclosure to parents and friends. Adolescence. 25(100), 959.-976.

Park SC \& Park YC (2020) Secondary emotional reactions to the Covid-19 outbreak should be identified and treated in Korea. Journal of Korean Medical Science. 35(17). DOI: 10.3346/JKMS.2020.35.E161.

Pfattheichera S, Nockurb L, Böhmc R, Sassenrathb C, Petersen MB (2020) The emotional path to action: Empathy promotes physical distancing and wearing face masks during the COVID-19 pandemic. DOI: 10.31234/osf.io/y2cg5.

Pierro A, Mannetti L, Converso D, Garsia V, Miglietta A, Ravenna M, Rubini M (1995) Caratteristiche strutturali della versione italiana della scala di bisogno di chiusura cognitiva. TPM. 2(3-4), 125-141.

Reich JW, Johnson LM, Zautra AJ, et al. (2006) Uncertainty of illness relationships with mental health and coping processes in fibromyalgia patients. Journal of Behavioral Medicine. 29, 307-316. DOI: 10.1007/s10865-006-9054-7.

Ritov I \& Baron J (1990) Reluctance to vaccinate: Omission bias and ambiguity. Journal of Behavioral Decision Making. 3(4), 263-277. DOI: 10.1002/bdm.3960030404.

Rosen NO \& Knäuper B (2009) A little uncertainty goes a long way: State and trait differences in uncertainty interact to increase information seeking but also increase worry. Health Communication. 24, 228-238. DOI: 10.1080/10410230902804125.

Rosen NO, Knäuper B \& Sammut J (2007) Do individual differences in intolerance of uncertainty affect health monitoring? Psychology and Health. 22(4), 413-430. DOI: 10.1080/14768320600941038.

Rubin G \& Wessely S (2020) The psychological effects of quarantining a city. The BMJ. 368. DOI: 10.1136/bmj.m313

Shigemura J, Ursano RJ, Morganstein JC, et al. (2020) Public responses to the novel 2019 coronavirus (2019-nCoV) in Japan: Mental health consequences and target populations. Psychiatry and Clinical Neurosciences. 74(4), 281. DOI: 10.1111/pcn.12988.

Tausczik Y, Faasse K, Pennebaker JW, et al. (2012) Public Anxiety and Information Seeking Following the H1N1 Outbreak: Blogs, Newspaper Articles, and Wikipedia Visits. Health Communication. 27(2), 179-185. DOI: $10.1080 / 10410236.2011 .571759$. 
Turner MM, Rimal RN, Morrison D, et al. (2006) The role of anxiety in seeking and retaining risk information: Testing the risk perception attitude framework in two studies. Human Communication Research. 32(2), 130-156. DOI: 10.1111/j.1468-2958.2006.00006.x.

Viscusi WK (1997) Alarmist decisions with divergent risk information. Economic Journal. 107(445), 16571670. DOI: 10.1111/j.1468-0297.1997.tb00073.x.

Viscusi WK, Magat WA and Huber J (1991) Communication of ambiguous risk information. Theory and Decision. 31(2-3), 159-173. DOI: 10.1007/BF00132991.

Wang C, Pan R, Wan X, et al. (2020) A longitudinal study on the mental health of general population during the COVID-19 epidemic in China. Brain, Behavior, and Immunity. 87, 40-48. DOI: 10.1016/j.bbi.2020.04.028.

Weber EU, Blais AR \& Betz NE (2002) A Domain-specific Risk-attitude Scale: Measuring Risk Perceptions and Risk Behaviors. Journal of Behavioral Decision Making. 1, 263-290. DOI: 10.1002/bdm.414.

Webster DM \& Kruglanski AW (1994) Individual Differences in Need for Cognitive Closure. Journal of Personality and Social Psychology. 67(6), 1049-1062. DOI: 10.1037/0022-3514.67.6.1049.

World Health Organization (WHO, 2020) Novel Coronavirus (2019-nCoV). Situation Report - 13. Available at: https://www.who.int/docs/default-source/coronaviruse/situation-reports/20200202-sitrep-13-ncovv3.pdf. (accessed May 2, 2020)

Zhao Y, Cheng S, Yu X, et al. (2020) Chinese public's attention to the COVID-19 epidemic on social media: Observational descriptive study. Journal of Medical Internet Research. 22(5), e18825. DOI: $10.2196 / 18825$.

\section{Table}

Table 1. Means, standard deviations, and correlations between main variables and control variables. 


\begin{tabular}{|c|c|c|c|c|c|c|c|c|c|c|}
\hline & $\mathrm{M}(\mathrm{SD})$ & (1) & (2) & (3) & $(4)$ & (5) & (6) & (7) & (8) & (9) \\
\hline Gender & - & $-.27 * * *$ & .05 & -.01 & -.09 & .10 & .05 & -.02 & $.16^{*}$ & $.26 * * *$ \\
\hline Age (1) & $\begin{array}{c}36.67 \\
(15.21)\end{array}$ & & -.09 & .07 & .11 & .07 & .02 & .09 & $-.16^{*}$ & -.04 \\
\hline Family size (2) & $2.49(1.41)$ & & & $-.16^{*}$ & $.21^{* *}$ & .03 & -.07 & -.01 & -.13 & -.09 \\
\hline COVID-19 exposure (3) & $.97(1.04)$ & & & & .05 & .02 & $.23 * * *$ & $.19 * * *$ & .03 & .03 \\
\hline Risk Aversion (4) & $3.8(.64)$ & & & & & .01 & .07 & $.21 * *$ & $.29 * * *$ & -.05 \\
\hline Intolerance for ambiguity (5) & $3.14(.40)$ & & & & & & .07 & .04 & -.05 & $.34 * * *$ \\
\hline Worry (6) & $3.67(.89)$ & & & & & & & $.20 * *$ & -.05 & $.34 * * *$ \\
\hline $\begin{array}{l}\text { Health-related information } \\
\text { seeking behavior ( } 7 \text { ) }\end{array}$ & $1.92(1)$ & & & & & & & & $.20 * *$ & $.15^{*}$ \\
\hline Utility of the lockdown (8) & $4.27(.75)$ & & & & & & & & & $.22^{* *}$ \\
\hline Distress (9) & $3.08(.39)$ & & & & & & & & & \\
\hline
\end{tabular}

Note: $* p<.05$; ** $p<.01 ; * * *<.001$; correlation coefficients are Pearson's $r$ except for ${ }^{1}$ Kendall's tau-b.

\section{Figures}

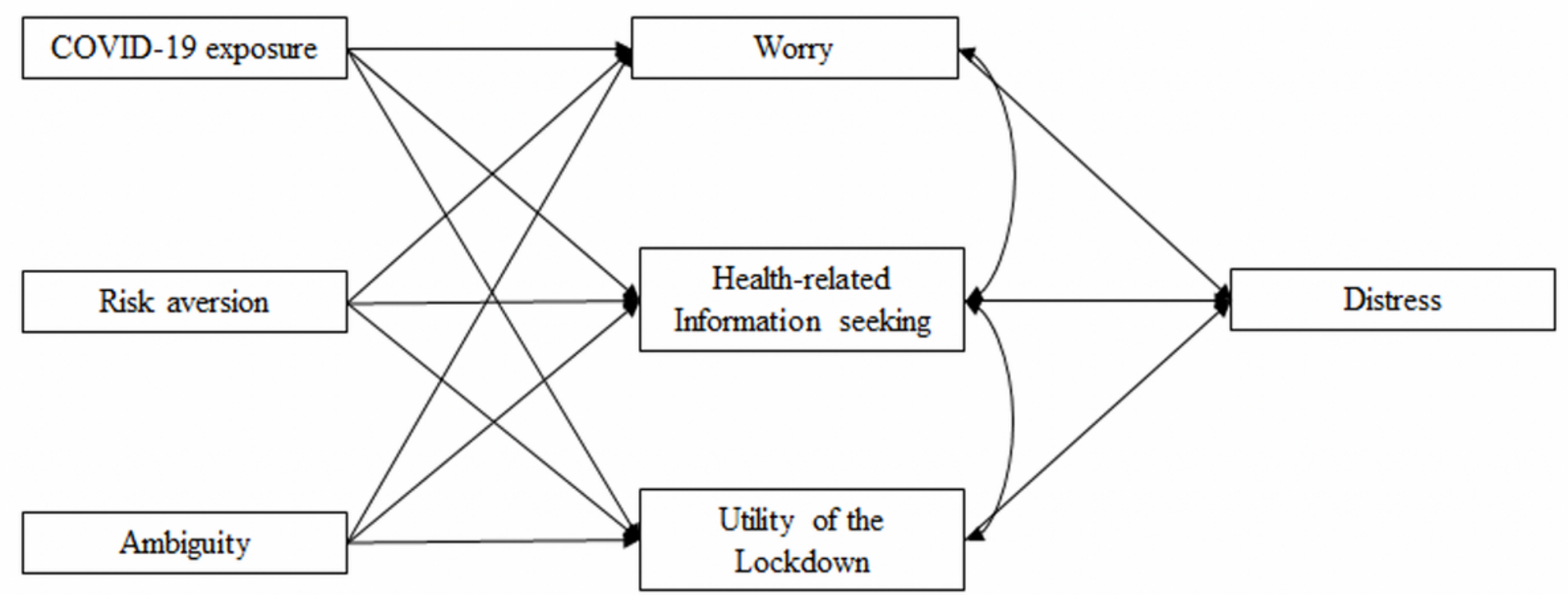

\section{Figure 1}

Theoretical model tested. 


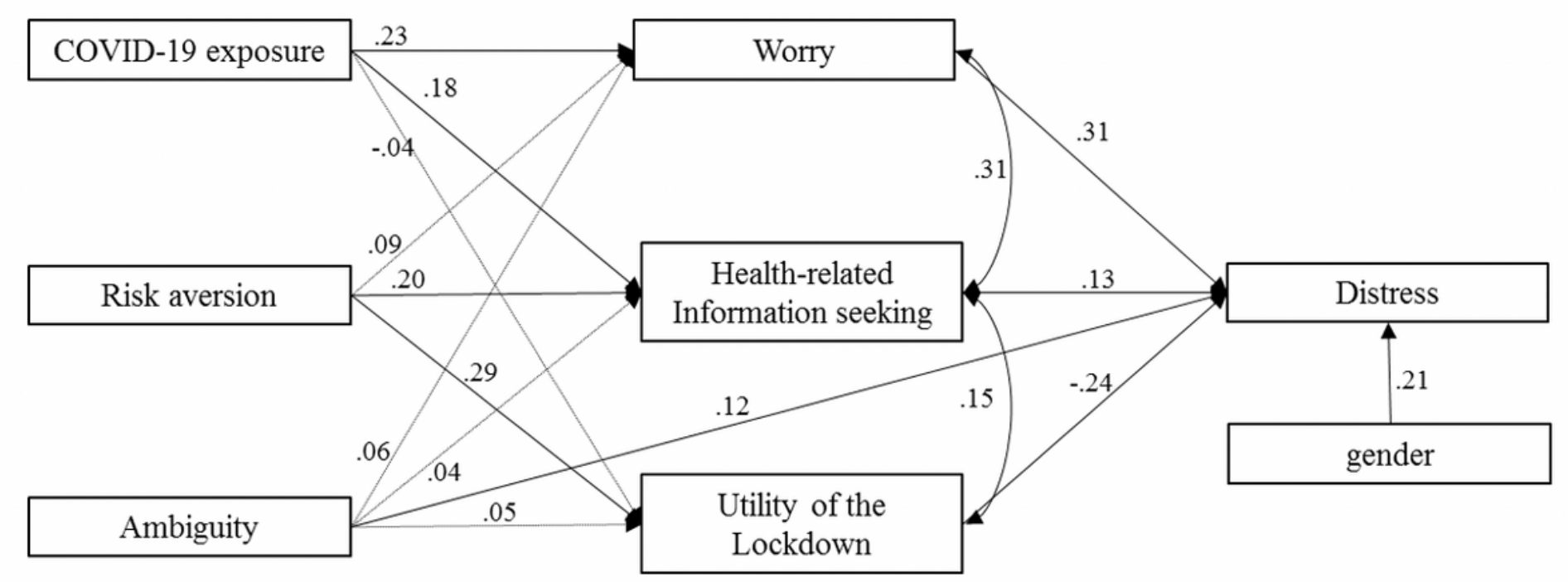

Figure 2

Results of the tested model. Dot lines indicate non-significant paths; plain lines indicate significant paths. 